\title{
EFEITOS DE INSETICIDAS BOTÂNICO E SINTÉTICOS SOBRE OVOS E LARVASDE CYCLONEDA SANGUINEA (LINNAEUS) (COLEOPTERA: COCCINELLIDAE) EM CONDIÇÕES DE LABORATÓRIO
}

\section{L.V. Cosme', G .A. Carvalho'², A .P. Moura ${ }^{3}$}

IUniversidade Federal deViçosa, Departamento deBiologia Animal, Setor de Entomologia, Av. P.H. Rolfs, s/ №, CEP 36571-000, Viçosa, MG, Brasil. E-mail: cosme@insecta.ufv.br

\section{RESUMO}

Este trabalho objetivou avaliar os efeitos do azadirachtina e de al guns inseticidas sintéticos utilizados nacultura do al godoei ro sobreovoselarvas deprimeiro equarto instares do predador Cycloneda sanguinea (Linnaeus). N im-I-Go ${ }^{\circledR}$ (azadirachtina) foi utilizado nas concentrações de $1 \%$, $5 \%$ e $10 \%$, correspondendo a $10 \mathrm{mg} / \mathrm{L}, 50 \mathrm{mg} / \mathrm{L}$ e $100 \mathrm{mg} / \mathrm{L}$ de azadirachtina, respectivamente. Os inseticidas utilizad os foram teflubenzurom ( $\mathrm{N} \mathrm{omolt} 150 \mathrm{SC}$ ) (0,15 g i.a./ L), I ambdacialotrina (Karate Zeon 250 CS) (0,08 g i.a./ L) e clorpirifós (Vexter 480 CE) (1,6 g i.a./ L). 0 tratamento testemunha foi composto somente por água destilada. Utilizaram-se sete tratamentos com seis repetições, sendo cada parcel aformada por trêsovos ou larvas deC . san guinea, sendo as aplicações realizadas com um pulverizador manual. Os bioensaios foram mantidos a $25 \pm 2^{\circ} \mathrm{C}$, UR de $70 \pm$ $10 \%$ e fotofase de $12 \mathrm{~h}$. Os insetos foram alimentados ad libitum com o pulgão-verde-do-sorgo Schizaphis graminum (Rondani). Todos o compostos avaliados reduziram a viabilidade de ovos. Lambdacial otrinaeclorpirifósforamaltamentetóxicos paralarvas de 1oe 40 instar deC. san guinea. Azadirachtina a 50 e $100 \mathrm{mg} / \mathrm{L}$ foi tóxico para larvas de quarto instar deC. sanguinea. Em função da baixa toxicidade apresentada, azadirachtina $10 \mathrm{mg} / \mathrm{L}$ e teflubenzuron apresentam as características mais favoráveis para uso em associação com C. sanguinea, para o controle de pragas na cultura al godoeira, dentre os compostos testados.

PALAVRAS-CHAVE: Azadirachtina, produtos fitossanitários, joaninha, seletividade.

\section{ABSTRACT}

EFFECTS OF BOTANICAL AND SYNTHETIC INSECTICIDES ON EGGSAND LARVAE OF CYCLONEDA SANGUINEA (LINNAEUS) (COLEOPTERA: COCCINELLIDAE) UNDER LATORATORY CONDITIONS. Theobjectiveof thiswork wasto evaluatetheeffects of azadirachtin and somesyntheticinsecticides used in thecotton crops on eggs and first- and fourth-instar larvae of Cycloneda sanguinea (Linnaeus). The neem-based insecticide Nim-I-Go ${ }^{T M}$ was used in the following concentrations: $1 \%$ (10 mg of azadirachtin/ L), $5 \%$ ( $50 \mathrm{mg}$ of azadirachtin/ $\mathrm{L}$ ) and $10 \%$ (100 mg of azadirachtin/ L). Thesyntheticinsecticides teflubenzuron (N omolt 150SC) (0.15ga.i./ L), lambdacyhal othrin (Karate Zeon $250 \mathrm{CS}$ (0.08 g a.i./ L) and chlorpyrifos (Vexter $480 \mathrm{CE}$ ) (1.6 $\mathrm{g}$ a.i./ L) al so were used in this study. Distilled water was used as control. A fully randomized experimental design wasused, with seven treatments and six replicates, each oneformed of three eggs or larvae of $\mathrm{C}$. sanguinea. A manual sprayer was used to spew the solutions. The bioassays were kept in a room at $25 \pm 2^{\circ} \mathrm{C}, \mathrm{RH}$ of $70 \pm 10 \%$ and $12 \mathrm{~h}$ photophase. The insects were fed with Schizaphis graminum (Rondani). Lambdacyhal othrin and chlorpyrifos were harmful to all stages of $\mathrm{C}$. sanguinea. Azadirachtin at 50 and $100 \mathrm{mg}$; $\mathrm{L}$ was toxic to fourth-instar larvae of $\mathrm{C}$. sanguinea. Based in its low toxicity, azadi rachtin $10 \mathrm{mg} / \mathrm{L}$ and teflubenzuron presentfavorablecharacteristics for use in the integrated pest management where $C$. sanguinea is an important factor of natural mortality of pests.

KEY WORDS: Azadirachtin, pesticides, lady-beetle, selectivity.

2Universidade Federal de Lavras, Departamento de Entomologia, Lavras, MG, Brasil.

3Universidade Federal Rural do Rio de Janeiro, Departamento de Genética, Seropédica, RJ, Brasil. 


\section{INTRODUÇÃO}

A preservação e manutenção de Inimigos N aturais (INs) nos agroecossistemas são imprescindíveis para o estabelecimento do controlebiológico natural, evitando-se efeitos indesejáveis como sel eção de populaçõesdeinsetos-pragaresistentesaosagrotóxicos, aparecimento de pragas secundárias e ressurgência de pragas. Permite redução na dependência de pesticidas, acarretando menor contaminaçãodo solo, água, faunaedo próprio homem, além dadiminuição dos custos de produção (Gravena, 2003). Deve-se salientar que, dentro dafilosofiado $M$ anejoI ntegrado de Pragas (MIP), o uso de agrotóxicos somente deve ser feito quando eles apresentarem algum tipo de seletividade (ReIS, 1996; HASSAN, 1997; Medina et al., 2001; Medina et al ., 2003).

Os insetos da família Coccinellidae apresentam grande importância no controle biológico, sendo que cerca de $90 \%$ dosinsetos pertencentesaessegrupo são considerados benéficos, em função de sua atividade predatória, principal mentedeafídeos eácaros (IPERGI, 1999). Dentre estes, a joaninha Cycloneda sanguinea (Linnaeus) (Coleoptera: Coccinellidae) apresenta-se como uma das espécies mais importantes no Brasil, predando inúmeras espécies de pulgões em diversos agroecossistemas. Umadasformas desepreservar esse inimigo natural, portanto, sefaz por meio dautilização de produtos seletivos (Medina et al ., 2003).

$\mathrm{N}$ a tentativa de se reduzir impactos ao ambiente causados por produtos sintéticos, vem crescendo o número de pesquisas com produtos naturais, sendo que a planta com atividade inseticida, A zadirachta indica A.Juss (M eliaceae), conhecida comumentepor nim, é considerada uma das mais importantes em várias partesdomundo (Medina etal.,2004). O óleo de nim apresenta efeitos colaterais para inúmeras espé cies de insetos-praga, como inibição da oviposição, da alimentação, do acasalamento, da motilidade intestinal, debiossíntese dequitina, ecausando mortalidade, sendotambémreferenciado naliteraturacomo um produto debaixa toxicidadea mamíferosealNs, etambém derápidadegradaçãonoambiente(BANKEN; Stark, 1997; Barbosa et al., 2000; Brunherotto, 2000; Brunherotto, Vendramim; 2001; Boeke et al., 2004; N ATHAN et al., 2005).

Destaforma, a compatibilização do uso do óleo de nim e de coccinelídeos no manejo de pragas surge comoumaestratégiaviável nocontextodeagricultura sustentável, porém, necessitando do desenvolvimento depesquisas para estudar tal associação. A ssim, o presentetrabalhotevecomo objetivoavaliar os efeitos do Nim-I-Go ${ }^{\circledR}$, uminseticidanatural àbasedo ól eo de nimedealguns inseticidas sintéticos recomendados para o controle de pragas na cultura do algodoeiro sobreC. sanguinea, em condições de laboratório.

\section{MATERIAL E MÉTODOS}

Criação de laboratório da presa. A espécie Schizaphis graminum (Rondani) (Hemiptera: A phididae) foi criada conforme metodologia proposta por pesquisadores do Centro Nacional de Pesquisas de Milho e Sorgo (CNPMS) - Embrapa (CRuz; Vendramim, 1989). Utilizaram-se folhas de sorgo da cultivar BR-301 retiradas de plantas cultivadas em uma área próxima ao Departamento de Entomologia da Universidade Federal de Lavras (UFLA).

Colônias de pulgões foram transferidas para seções foliares de sorgo com aproximadamente $63 \mathrm{~cm}^{2}$, dispostas em bandejas plásticas ( $40 \mathrm{~cm}$ de comprimento $\times 20 \mathrm{~cm}$ delargura $10 \mathrm{~cm}$ dealtura). Dentro de cada bandeja foi colocada uma placa de isopor de 36 $\mathrm{cm}$ de comprimento $\times 16 \mathrm{~cm}$ de largura $\times 1 \mathrm{~cm}$ de espessura contendo 18 orifícios de $4 \mathrm{~cm}$ de diâmetro cada (três fileiras de furos de $4 \mathrm{~cm}$ de diâmetro, seis furos por fileira), onde as seções foliares foram fixadas. Adicionou-se água até a metade do volume de cada bandeja, para permitir a conservação do material vegetativo por maior tempo. Cerca de duas a três vezes por semana as seções foliares foram trocadas, sendo colocadas ao lado das novas para ocorrência da migração dos pulgões. A criação foi mantida em sal a climatizadaa $25 \pm 2^{\circ} \mathrm{C}$, UR de $70 \pm 10 \%$ efotofase de $12 \mathrm{~h}$.

Criação de laboratório deC. sanguinea. O predadorfoi coletadoem plantas delaranjadacultivar Pêra Rio no pomar do Campus da UFLA, e criado em recipientes de vidro com $30 \mathrm{~cm}$ de comprimento $\times 30$ $\mathrm{cm}$ de largura $\times 30 \mathrm{~cm}$ de al tura, sendo fechadas na partesuperior com tecidotipovoil emantidosemsala climatizada regulada a $25 \pm 20$ C, UR de $70 \pm 10 \%$ e fotofase de $12 \mathrm{~h}$.

Diariamente, foram fornecidos pulgões $S$. graminumao predador, eretirados osseusovoscom auxílio depincel umedecido, os quais foram colocados em placas de Petri de $15 \mathrm{~cm}$ de diâmetro, fechadas com filme de cloreto de polivinila (PVC) e mantidas nas mesmas condições anteriormentecitadas. Ao eclodirem, as larvas foram transferidas para bandejas plásticas com $40 \mathrm{~cm}$ de comprimento $x 20 \mathrm{~cm}$ de largura $x 10 \mathrm{~cm}$ de al tura, na proporção de 25 larvas por bandeja. Estas bandejas continham seções foliares de sorgo da cultivar BR-301, de $63 \mathrm{~cm}^{2}$, infestadas com pul gões, permitindo que as larvas se alimentassemad libitum. A pósoconsumo total dos pulgões pelo predador realizou-se a substituição das seções foliares. Quando os coccinelídeos atingiram o estágio de pupa foram, então, transferidos para placas dePetri de $15 \mathrm{~cm}$ de diâmetro, onde permaneceram até a emergência dos adultos, os quais foram distribuídos em novos 
recipientes de vidro, dando início a outro ciclo de criação. A cada mês, realizaram-se coletas deadultos deC. sanguinea no Campus daUFLA, queforam incorporad os à criação delaboratório, evitando-se assim problemas de endogamia e degeneração genética da população.

Bioensaios. O produto comercial $\mathrm{Nim}-\mathrm{I}-\mathrm{Go}^{\circledR}$ foi utilizado nas concentrações de $1 \%$, 5\% e 10\%. Avaliaram-se também os inseticidas sintéticos teflubenzurom ( omolt150SC) (0,15gi.a./ L deágua), lambdacial otrina (KarateZeon $250 \mathrm{CS})(0,08$ gi.a./ L) e clorpirifós (Vexter 480 CE) (1,6 g i.a./ L), que foram aplicados nas maiores dosagens recomendadas pelosfabricantesparao controledepragasnaculturado algodoeiro. $\mathrm{N} \mathrm{im}-\mathrm{I}-\mathrm{Go}^{\circledR}$, segundo o seu fabricante, contém 1.000 mg deazadirachtina/ $L$ do produto comercial, sendo este considerado o principal ingrediente ativo de extratos denim (MedinA et al., 2004). O tratamento testemunha foi constituído somente de água destilada.

A plicação dos produtos. As pulverizações dos compostos foram realizadas por meio deum pulverizador manual com capacidadepara $600 \mathrm{~mL}$, assegurando a aplicação de 1,5 $\pm 0,5 \mathrm{mg}$ de calda química/ $\mathrm{cm}^{2}$, conforme metodologia estabelecida por membrosda IOBC (HASSAN, 1997). A cal ibração dos pulverizadores foi realizada aplicando-se água destilada em discos de papel-filtro que foram pesados antes e após as aplicações. A média das diferenças entre as pesagens foi utilizada como valor padrão. Os pulverizadoresforamaferidospreviamenteàmontagemde cada bioensaio.

Efeitos dos produtos sobre ovos e larvas de C. sanguinea. Ovoselarvas deprimeiro equartoinstares do predador foram obtidos da criação de laboratório etransferidosparaplacas dePetri de $15 \mathrm{~cm}$ dediâmetrocomauxílio deum pincel umedecido, ondereceberam os produtos.

No experimento com ovos, duas horas após a aplicação dos compostos, el es foram individualizadosem placas dePetri de $5 \mathrm{~cm}$ dediâmetro, queforam fechadas com filme plástico de PVC laminado. A pós a eclosão, as larvas sobreviventes foram colocadas sobre seções foliares de sorgo contendo pulgões em copos plásticos com capacidade para $50 \mathrm{~mL}$, onde permaneceram até o final do experimento. Os copos contendoasfolhas desorgo comospulgõeseaslarvas sobreviventes foram distribuídos em placas de Petri de15cm dediâmetro, emcujasbordas aplicou-seuma mistura de talco com fluon (politetrafluoroetileno) para impedir a fuga das larvas e mantidos em sala climatizada a $25 \pm 2^{\circ} \mathrm{C}$, UR de $70 \pm 10 \%$ efotofase de $12 \mathrm{~h}$.

Os efeitos dos compostos para larvas deprimeiro e quarto estádios foram avaliados conforme metodologia descrita por BANKEN; STARK (1997). N es- ses bioensaios, após aplicação dos produtos sobre indivíduos de primeiro equarto instares, estes foram transferidos para seções foliares de sorgo contendo pulgões que encontravam-se em copos plásticos, de forma semelhante à realizada para o experimento comovos. A pós puparem, osinsetosforam colocados em placas de Petri de $5 \mathrm{~cm}$ de diâmetro, que foram fechadas com filmedeplástico dePVC laminado, até a emergência dos adultos.

A valiaram-se diariamente a viabilidade dos embriões e das larvas e também a duração de cada estádioe/ ou fase. N obioensaio comlarvas deprimeiroinstar também foram determinadasas razõessexuais.

Foi utilizado o delineamento experimental inteiramente casualizado com sete tratamentos e seis repetições, sendo cada parcela constituída por três indivíduos (ovos ou larvas de primeiro e quarto instares).

A nálises estatísticas. Os dados obtidos foram submetidos àanálise devariância através do programaestatísticoSisvar (FerReIRA, 2000), sendoasmédias dos tratamentos comparadas por meio do teste de agrupamento de Scott-Knott a 5\% de significância (ScotT;KNotT, 1974). Real izou-seanálisederegressão paraosdadosreferentesàsobrevivênciadaslarvasde primeiro instar eda duração do quarto estádio, quando azadirachtina foi aplicada em larvas de primeiro instar.

\section{RESULTADOS}

Efeitos dos produtos sobre ovos. Nenhum dos produtos avaliados afetou a duração do período embrionário, do primeiro esegundo instares (Tabela 2), nem a sobrevivência dos dois primeiros estádios larvaisquando foramaplicadossobreovos (Tabela1). Contudo, a viabilidade dos ovos foi reduzida por todos os compostos utilizados, principalmente pelo inseticida lambdacialotrina, que provocou mortalidade de todos os embriões (Tabela 1). Independente da concentração utilizada, azadirachtina foi nociva aos embriões do predador, sendo seus efeitos tão prejudiciaisquanto aosobservados paraclorpirifóse teflubenzurom (Tabela 1).

Efeitos dos produtos sobre larvas de primeiro estádio. Quando aplicado sobre larvas de primeiro instar de $\mathrm{C}$. sanguinea, teflubenzurom causou diminuição na sobrevivência das Iarvas no tercei ro instar (Tabela 3); entretanto, não reduziu a duração dos estádios Iarvais (Tabela 4). Clorpirifós elambdacial otrina foram os compostos mais nocivos às larvas, provocando mortal idades de 100 e $70 \%$ mortalidade logo após as aplicações (Tabela 3). 


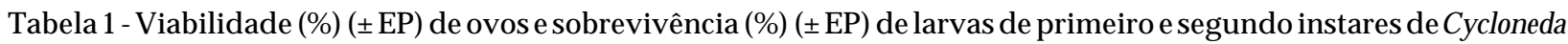
sanguinea provenientes de ovos tratados. Temperatura de $25 \pm 2^{\circ} \mathrm{C}$, UR de $70 \pm 10 \%$ e fotofase de $12 \mathrm{~h}$.

\begin{tabular}{|c|c|c|c|}
\hline \multirow[t]{2}{*}{ Tratamentos } & \multirow[t]{2}{*}{ Ovos 2,3} & \multicolumn{2}{|c|}{ Fase larval $^{1}$} \\
\hline & & 1o Instar & 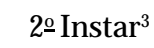 \\
\hline Azadirachtina 10 mg/ L & $52,8 \pm 0,05 b$ & $92,8 \pm 5,97$ & $80,0 \pm 0,03$ \\
\hline Azadirachtina 50 mg/ L & $41,7 \pm 0,06 b$ & $91,7 \pm 8,33$ & $73,9 \pm 0,06$ \\
\hline Azadirachtina $100 \mathrm{mg} / \mathrm{L}$ & $44,4 \pm 0,04 b$ & $83,3 \pm 10,54$ & $65,3 \pm 0,04$ \\
\hline Clorpirifós & $41,7 \pm 0,05 b$ & $79,2 \pm 10,92$ & $75,0 \pm 0,14$ \\
\hline Lambdacialotrina & $0,0 \pm 0,00 c$ & - & - \\
\hline Teflubenzurom & $33,3 \pm 0,10 b$ & $95,0 \pm 4,08$ & $66,7 \pm 0,05$ \\
\hline Testemunha & $69,4 \pm 0,02 \mathrm{a}$ & $91,7 \pm 5,27$ & $94,4 \pm 0,02$ \\
\hline Teste F & $19,558^{\text {*k }}$ & $0,620^{\text {ns }}$ & $0,675^{\text {ns }}$ \\
\hline CV (\%) & 25,82 & 21,75 & 22,76 \\
\hline
\end{tabular}

${ }^{1}$ As médias não diferem entre si pelo teste $F$ a $5 \%$ de significância.

${ }^{2}$ Médias seguidas das mesmas letras não diferem entre si pelo teste de Scott-Knott a 5\% de significância.

${ }^{3}$ Dados transformados para arcsen $\sqrt{(x / 100)}$.

Tabela2-Duração (dias) ( \pm EP) do período embrionárioe do primei ro esegundo instaresdelarvas deC yclon edasanguinea provenientes de ovos tratados. Temperatura de $25 \pm 2^{\circ} \mathrm{C}$, UR de $70 \pm 10 \%$ e fotofase de $12 \mathrm{~h}$.

\begin{tabular}{lccr}
\hline Tratamentos & Período embrionário ${ }^{1}$ & \multicolumn{2}{c}{ Fase larval $^{1}$} \\
\cline { 3 - 4 } & & 1oInstar & 2 Instar ${ }^{2}$ \\
\hline Azadirachtina 10 mg/ L & $2,9 \pm 0,07$ & $1,9 \pm 0,05$ & $1,6 \pm 0,02$ \\
Azadirachtina 50 mg/ L & $2,9 \pm 0,04$ & $2,0 \pm 0,14$ & $1,3 \pm 0,06$ \\
Azadirachtina 100 mg/ L & $2,8 \pm 0,05$ & $2,0 \pm 0,04$ & $1,5 \pm 0,01$ \\
Clorpirifós & $2,8 \pm 0,04$ & $2,3 \pm 0,36$ & $1,5 \pm 0,01$ \\
Lambdacialotrina & $*$ & $*$ & $*$ \\
Teflubenzurom & $2,8 \pm 0,06$ & $1,7 \pm 0,12$ & $2,3 \pm 0,03$ \\
Testemunha & $2,9 \pm 0,08$ & $1,5 \pm 0,16$ & $1,6 \pm 0,01$ \\
\hline TesteF & $1,402^{\text {ns }}$ & 2,179 ns & $1,312^{\text {ns }}$ \\
CV (\%) & 5,13 & 15,38 & 15,97 \\
\hline
\end{tabular}

${ }^{1}$ As médias não diferem entre si pelo teste $F$ a $5 \%$ de significância.

${ }^{2}$ Dados transformados para $\sqrt{(x+0,5)}$.

*N úmero de indivíduos insuficiente para avaliação do parâmetro.
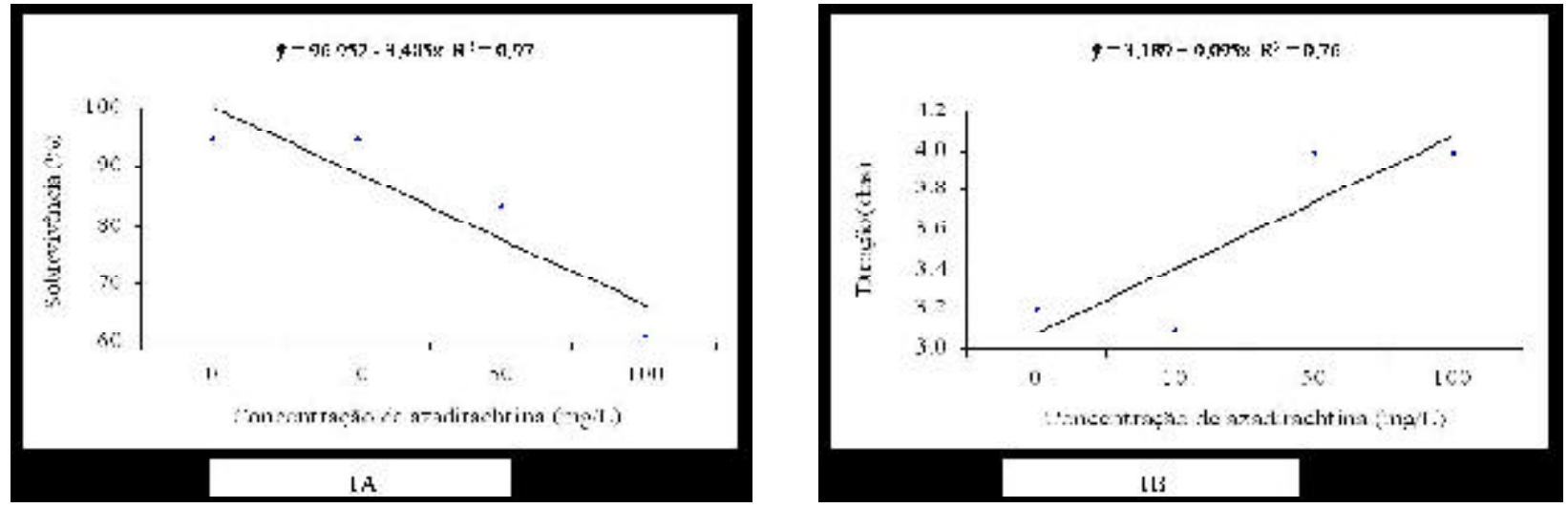

Fig. 1 - Modelos deregressão para sobrevivência de larvas de primei ro instar deCycloneda sanguinea (1A) eduração do quarto instar (1B) quando larvas foram tratadas com azadirachtina. 
Tabela 3-Sobrevivência (\%) ( \pm EP) delarvas e pupas de Cycloneda sanguinea provenientes de larvas de primeiro instar tratadas. Temperatura de $25 \pm 2^{\circ} \mathrm{C}$, UR de $70 \pm 10 \%$ e fotofase de $12 \mathrm{~h}$.

\begin{tabular}{|c|c|c|c|c|c|}
\hline \multirow[t]{2}{*}{ Tratamentos } & \multicolumn{4}{|c|}{ Fase larval } & \multirow[t]{2}{*}{ Fasepupal } \\
\hline & 1ㅇ Instar ${ }^{2,3}$ & 2ㅇ Instar ${ }^{1}$ & 30 Instar 2 & 40 Instar ${ }^{1}$ & \\
\hline Azadirachtina $10 \mathrm{mg} / \mathrm{L}$ & $94,4 \pm 3,51 \mathrm{a}$ & $83,8 \pm 6,23$ & $89,2 \pm 4,90 a$ & $100,0 \pm 0,00$ & $94,4 \pm 5,56$ \\
\hline Azadirachtina $50 \mathrm{mg} / \mathrm{L}$ & $83,3 \pm 4,30 a$ & $96,7 \pm 3,33$ & $97,2 \pm 2,78 a$ & $96,7 \pm 3,33$ & $88,3 \pm 8,33$ \\
\hline Azadirachtina $100 \mathrm{mg} / \mathrm{L}$ & $61,1 \pm 5,56 b$ & $86,9 \pm 6,09$ & $95,8 \pm 4,17 a$ & $88,9 \pm 7,03$ & $95,8 \pm 4,17$ \\
\hline Clorpirifós & $0,0 \pm 0,00 c$ & - & - & - & - \\
\hline Lambdacial otrina & $30,0 \pm 0,00 c$ & $*$ & $*$ & * & * \\
\hline Teflubenzurom & $77,8 \pm 8,24 a$ & $95,8 \pm 3,27$ & $76,4 \pm 10,19 b$ & $100,0 \pm 0,00$ & $97,2 \pm 2,78$ \\
\hline Testemunha & $94,4 \pm 3,51$ a & $96,7 \pm 3,33$ & $100,0 \pm 0,00 a$ & $97,2 \pm 2,78$ & $96,7 \pm 3,33$ \\
\hline Teste F & $85,652^{* *}$ & $1,718^{\text {ns }}$ & $2,921^{* *}$ & $1,520^{\text {ns }}$ & $0,475^{\text {ns }}$ \\
\hline CV (\%) & 18,78 & 12,42 & 14,77 & 9,37 & 13,54 \\
\hline
\end{tabular}

${ }^{1}$ As médias não diferem entre si pelo teste $\mathrm{F}$ a $5 \%$ de significância.

${ }^{2}$ Médias segui das das mesmas letras não diferem entre si pelo teste de Scott-Knott a 5\% de significância.

${ }^{3}$ Dados transformados para arcsen $\sqrt{(x / 100)}$.

*Número de indivíduos insuficiente para avaliação do parâmetro.

Tabela 4 - Duração (dias) dos estádios larvais e de pupas e razão sexual ( $\pm E P$ ) deCycloneda sanguinea quando larvas de primeiro instar foram tratadas. Temperatura de $25 \pm 2^{\circ} \mathrm{C}$, UR de $70 \pm 10 \%$ e fotofase de $12 \mathrm{~h}$.

\begin{tabular}{|c|c|c|c|c|c|c|}
\hline \multirow[t]{2}{*}{ Tratamentos } & \multicolumn{4}{|c|}{ Fase larval } & \multirow[t]{2}{*}{ Fase pupal $^{1}$} & \multirow[t]{2}{*}{ Razãosexual } \\
\hline & 1ㅇ Instar ${ }^{1}$ & 20 Instar ${ }^{1,3}$ & 30. Instar ${ }^{1,3}$ & $4^{\circ}$ Instar $^{2}$ & & \\
\hline Azadirachtina $10 \mathrm{mg} / \mathrm{L}$ & $1,9 \pm 0,22$ & $1,7 \pm 0,08$ & $1,6 \pm 0,11$ & $3,1 \pm 0,06 a$ & $3,6 \pm 0,20$ & $0,5 \pm 0,05$ \\
\hline A zadirachtina $50 \mathrm{mg} / \mathrm{L}$ & $1,7 \pm 0,05$ & $1,3 \pm 0,02$ & $1,5 \pm 0,03$ & $4,0 \pm 0,11 b$ & $3,2 \pm 0,15$ & $0,5 \pm 0,02$ \\
\hline A zadirachtina $100 \mathrm{mg} / \mathrm{L}$ & $1,9 \pm 0,15$ & $1,3 \pm 0,02$ & $1,5 \pm 0,06$ & $4,0 \pm 0,20 b$ & $3,3 \pm 0,16$ & $0,5 \pm 0,07$ \\
\hline Teflubenzurom & $1,6 \pm 0,03$ & $1,7 \pm 0,07$ & $1,7 \pm 0,08$ & $2,8 \pm 0,17 a$ & $3,6 \pm 0,20$ & $0,6 \pm 0,05$ \\
\hline Testemunha & $1,7 \pm 0,02$ & $1,3 \pm 0,01$ & $1,3 \pm 0,04$ & $3,2 \pm 0,13 a$ & $3,3 \pm 0,13$ & $0,5 \pm 0,04$ \\
\hline Teste F & $0,897^{\text {ns }}$ & $1,913^{\text {ns }}$ & $0,548^{\text {ns }}$ & 14,972 ** & $1,651^{\text {ns }}$ & $0,068^{\text {ns }}$ \\
\hline CV (\%) & 16,92 & 8,63 & 11,99 & 10,25 & 12,15 & 11,93 \\
\hline
\end{tabular}

${ }^{1}$ As médias não diferem entre si pelo teste $\mathrm{F}$ a $5 \%$ de significância.

${ }^{2}$ M édias seguidas das mesmas letras não diferem entre si pelo teste de Scott-Knott a 5\% de significância.

3Dados transformados para $\sqrt{(x+0,5)}$.

Tabela 5 - Sobrevivência (\%) eduração (dias) ( \pm EP) delarvas dequarto instar ede pupas deC yclon eda sanguinea, quando larvas de quarto instar foram tratadas. Temperatura de $25 \pm 2^{\circ} \mathrm{C}$, UR de $70 \pm 10 \%$ e fotofase de $12 \mathrm{~h}$.

\begin{tabular}{|c|c|c|c|c|}
\hline \multirow[t]{2}{*}{ Tratamentos } & \multicolumn{2}{|c|}{ Quarto instar } & \multicolumn{2}{|c|}{ Fase pupal } \\
\hline & Sobrevivência ${ }^{2}$ & $\overline{\text { Duração }^{1}}$ & 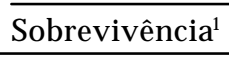 & $\overline{\text { Duração }^{1}}$ \\
\hline Azadirachtina $10 \mathrm{mg} / \mathrm{L}$ & $90,0 \pm 4,47 a$ & $3,5 \pm 0,16$ & $96,7 \pm 0,76$ & $4,7 \pm 0,21$ \\
\hline Azadirachtina $50 \mathrm{mg} / \mathrm{L}$ & $40,0 \pm 10,33 b$ & $*$ & $*$ & $*$ \\
\hline A zadirachtina 100 mg/ L & $36,7 \pm 6,15 b$ & * & * & * \\
\hline Clorpirifós & $0,0 \pm 0,0 c$ & - & - & - \\
\hline Lambdacialotrina & $0,0 \pm 0,0 c$ & - & - & - \\
\hline Teflubenzurom & $90,0 \pm 4,47 a$ & $3,4 \pm 0,13$ & $93,2 \pm 0,76$ & $4,8 \pm 0,17$ \\
\hline Testemunha & $86,7 \pm 6,67 a$ & $3,1 \pm 0,08$ & $95,0 \pm 0,76$ & $4,3 \pm 0,21$ \\
\hline Teste F & $50,032^{* *}$ & $3,188^{n s}$ & $2,056^{\text {ns }}$ & $1,667^{\text {ns }}$ \\
\hline CV (\%) & 28,56 & 9,37 & 3,15 & 10,48 \\
\hline
\end{tabular}

${ }^{1}$ As médias não diferem entre si pelo teste $\mathrm{F}$ a $5 \%$ de significância.

${ }^{2}$ As médias seguidas das mesmas letras não diferem entre si pelo teste de Scott-Knott a 5\% de significância.

*Número de indivíduos insuficiente para avaliação do parâmetro. 
A zadirachtina, na maior dosagem, reduziu a sobrevivência das larvas de primeiro estádio, com mé dia de $61,1 \%$ (Tabela 3 ), e para aquelas de quarto instar provenientes dessas, esse produto, nas dosagens de 50 e $100 \mathrm{mg} / \mathrm{L}$, provocou um prolongamento do último instar larval, com médias de a 4,0 dias (Tabela 4). Verificou-se que houve correlação linear inversa entre as concentrações do óleo de nim e a sobrevivência das larvas de primeiro instar, e direta entreaduração do quarto estádiolarval eas dosagens (Fig. 1). Nosdemaisestádioslarvais enafasedepupa não houve efeito significativo da azadirachtina. A razão sexual não foi afetada por nenhum dos tratamentos avaliados (Tabela 4). Verificou-se, também, a presença delarvas com anomalias morfológicas nos tratamentos com o óleo de nim.

Efeitos dos produtos sobre larvas dequarto estádio.Clorpirifóselambdacial otrinaprovocaram $100 \%$ demortalidade das larvas de quarto instar imediatamenteapós a pulverização; já teflubenzurom foi inócuo, não afetando nenhumadascaracterísticas biológicas avaliadas. Azadirachtina 50 e $100 \mathrm{mg} / \mathrm{L}$ reduziram a sobrevivência das larvas de quarto estádio; porém, na concentração de $10 \mathrm{mg} / \mathrm{L}$ ele mostrou-se inócuo (Tabela 5).

\section{DISCUSSÃO}

Alguns inibidores da síntese de quitina podem impedir a síntese desse polissacarídeo no embrião, uma vez que atuam na polimerização de unidades UDP-N -acetil-D-glucosamino, o último precursor da quitina durante sua síntese (Izawa et al., 1985). Atribui-seaissoa mortalidadeembrionária deC .sanguinea verificada para teflubenzurom. As larvas eclodidas dos ovos tratados não apresentaram problemas no seu desenvolvimento possivelmente devido à metabolização e/ ou excreção do produto pelo seu organismo. Para clorpirifós, observou-sequea maioria dos ovos ficou escura, porém houve baixa viabilidade, indicando que a morte dos embriões ocorreu próximo à eclosão das larvas.

A redução daviabilidadedosovosocasionadapela azadirachtinademonstraquealgumdeseusingredientes ativos, possivelmente a azadirachtina, interfere no desenvolvimento embrionário. Já foi demonstrado que aazadirachtina afetaas mitocôndrias, podendo deixálasinoperantesesemfunção ( MORDUE; BLACKWELL, 1993; VoGt etal., 1998). Como asmitocôndrias são as responsáveispelaproduçãodeenergianecessáriaaodesenvolvimento dos embriões, é provável que tenham sido afetadas. A açãoovicidadoóleodenimparacoleópteros também foi observada por CREDLAND (1992) em insetos dafamíliaBruchidae. Segundoesseautor, apresençade umpequenofunil (micrópila) nofinal daparteposterior do ovo pode ter facilitado a entrada do óleo de nim, provocandoamortedoembrião.Aslarvasoriundasdos ovos tratados não foram afetadas pela azadirachtina, devido, provavelmente, àmetabolização e/ ou excreção do produto no corpo do inseto.

As anormalidades morfológicas observadas nas larvas, principalmente quando a azadirachtina foi aplicadasobreaquel as deprimeiro estádio, jáforam descritas por Banken; STARK (1997) em Coccinella septempunctata Linnaeus (Coleoptera: Coccinellidae), quando eles real izaram pulverizações diretas sobre larvas de primeiro e quarto instares. Além desse efeito, produtosàbasedenimpodem provocar deformação das asas quando aplicado nas formas jovens deC. septempunctata. Deacordo com esses autores, o efeito do óleo denimédependentedesua dosagem, o que foi confirmado no presente trabal ho quando larvas de primeiro instar de C. sanguinea foram tratadas, visto que houve correlação linear inversa entre a sobrevivência das larvas e direta para duração do quarto estádio com a dosagem da azadirachtina.

A mortalidade causada pela azadirachtina para larvas de primeiro e quarto instares pode ser atribuída, segundo BANKen; Stark (1998), ao momento de introdução da azadirachtina nos ciclos hormonais do inseto eà meia-vida do produto após a absorção. Duranteafaselarval doinsetooecdisônioeohormônio juvenil são produzidos, sendo que a sua concentração pode ser alterada sensivelmente. Contudo, no último instar, a quantidade do hormônio juvenil decresce, permitindo que o nível de ecdisônio aumente provocando a pupação em insetos holometabólicos. A azadirachtinainterfereno equilíbrio existenteentre os dois hormônios. Esses autores relataram que uma alteração dos níveis de ecdisteróides antes da metamorfose parece ser mais crítica que a ação da azadirachtina nos instares iniciais, possivelmente porqueas larvas jovens sofrem os efeitos dessa substância antes da pupação. Este fato foi verificado no presente trabalho, uma vez que as larvas tratadas sobreviventes apresentaram em sua maioria um desenvolvimento normal.

O prolongamento do último instar, observado para larvas de primeiro estádio tratadas com azadirachtina $100 \mathrm{mg} / \mathrm{L}$, corrobora os resultados de BANKEN; StARK (1997). Provavelmente, as larvas não conseguiram metabolizar todo o produto antes de puparem. Esse efeito também pode estar relacionado ao fato da azadirachtinaatuarnosprocessosdetrocadetegumento ereduçãodaal imentaçãodosinsetos(VENDRAMIM , 1997; BARBOSA et al., 2000; BRUNHEROTTO, 2000).

Outrosestudos demonstraram quea aplicação de produtosou extratos denimprovocou mortalidadede coccinelídeos e crisopídeos. LoWERY; IsMAN (1995) verificaram que emulsões de nim reduziram a sobrevi- 
vência do coccinelídeo Coccinella undecimpunctata Linnaeus (Coleoptera: Coccinellidae). Para o crisopídeo Chrysoperla carnea (Stephens) (N europtera: Chrysopidae), VoGt et al . (1998) relataram quea aplicaçãodeformulações denimemlaboratório provocou alterações na cutícula e múscul os dos insetos tratados, mandíbulas mal formadas, desorientação das microfibrilas, destruição das mitocôndrias e fibras musculares mais espaçadas, além de mortalidade.

Os testes com óleo de nim sobre organismos não alvos, segundo BANKEN; STARK (1998), devem ser realizadosutilizando-sediferentesformas deexposição dos organismos ao produto para se conhecer o seu real impacto. Entretanto, alguns autores relataram queprodutosàbasedenimjápodemserutilizadosem programas demanejo integrado de pragas por serem seletivos a diversos inimigos naturais (GRIPWALL, 1999). Os efeitos nocivos de lambdacialotrina e clorpirifós já foram avaliados para os coccinelídeos predadores C. sanguinea e Eriopsis connexa Germar (Coleoptera: Coccinellidae) e para parasitóides A phidius spp. (Hemiptera: A phididae). Os produtos foram classificados como altamente tóxicos aos predadores, seguindo a seguinte escala: $\mathrm{S}$ (seletivo) $=0$ $20 \%$ de mortalidade, $\mathrm{B}$ (baixa) $=21-40 \%$, M (média) $=$ 41-60\%, A (alta) $=61-100 \%$ (LHAMBY; BACALTCHUK, 2007). Esses efeitos também foram constatados no presente trabal ho, sendo que os inseticidas impediram o desenvolvimento normal do predador, matando osinsetos atémesmo em poucosminutosapóssua aplicação, pois ambos agem em nível de sistema nervoso central.

$\mathrm{N}$ a presente pesquisa, foi constatado que $\mathrm{N} \mathrm{im-I-}$ $\mathrm{Go}^{\circledR}$ foi prejudicial a C. sanguinea, em condições de laboratório, demonstrando que os inseticidas naturais podem ser tão tóxicos a IN s quanto os si ntéticos. LOWERY; ISMAN (1995) constataram que, mesmo em condições de campo, o óleo denim ainda apresentou toxicidade a outros IN s de pulgões. Dessa forma, torna-se necessária a realização de testes em condições de semicampo e campo para averiguação dos efeitos do $\mathrm{N} \mathrm{im}-\mathrm{I}-\mathrm{Go}^{\circledR}$ sobre este predador.

\section{CONCLUSÕES}

- Azadirachtina a $10 \mathrm{mg} / \mathrm{L}$ eteflubenzuron $(0,15$ $\mathrm{g} / \mathrm{L}$ ) apresentam características favoráveis para uso no manejo integrado depragas ondeC. sanguineaéum importante fator de mortalidade natural de insetospraga;

- Azadirachtina a 50 e $100 \mathrm{mg} / \mathrm{L}$ apresenta de moderada a alta toxicidade a essa espécie de coccinelídeo;

- Lambdacialotrina $(0,08 \mathrm{~g} / \mathrm{L})$ éaltamentetóxico para ovos deC. sanguinea;
- Clorpirifós(1,6gi.a./ L) elambdacial otrina(0,08 g/ L) sãotóxicosalarvas deprimeiro equartoinstares deC. sanguinea;

-Todosostratamentosreduziramaviabilidadede ovos.

\section{AGRADECIMENTOS}

A o CN Pq pela colaboração financei ra no fornecimento da bolsa deiniciação científica ( $\mathrm{PIBIC/CNPq}$ ) ao primeiro autor.

REFERÊNCIAS

BANKEN, J.A.O.; STARK, J.D. Stage and age influence on the susceptibility of Coccinella septempunctata (Coleoptera: Coccinellidae) after direct exposure to Neemix, a Neem insecticide. J ournal of E conomic Entomol ogy, v.90, n.5, p.1103-1105, 1997.

BANKEN, J.A.O.; STARK, J.D. Multiple routes of pesticide exposure and the risk of pesticides to biological controls: a study of neem and the sevenspot lady beetle, Coccinella septempunctata $L$. Journal of E conomic Entomology, v.91, n.1, p.1-6, 1998.

Barbosa, A.P.; Ambrosano, E.J.; Abreu Júnior, H. Nim: o protetor natural múltiplo. Instituto Agronômico de Campinas, Campinas. 2000. 41p.

Boeke,S.J.;Boersma, M.G.;A LINK, G.M.;V AN LoON,J.J.A .;V AN HuIS,A.;D ICKE, M.;RIETJEns, I.M.C.M.Safety evaluation of neem (A zadirachtaindica) derived pesticides.J ournal of Ethnopharmacology, v.94, p.25-41, 2004.

BRUnherotto, R. Bioatividade de extratos aquosos de M elia azedarach L. eA zadirachtaindica A .J uss (M eliaceae) sobre Tuta absoluta (M eyrick, 1917) (Lep.: G elechiidae) criadas em diferentes genóti pos detomatei ro. 2000, 76p. Dissertação (Mestrado emEntomologia) - EscolaSuperior de Agricultura “Luiz deQueiroz"- UniversidadedeSão Paulo, Piracicaba, 2000.

BRUnherotTo, R.; VendRAmim, J.D. Bioatividadedeextratos aquosos deM eliaazedarach L. sobreo desenvolvimento deT utaabsoluta (Meyrick) (Lepidoptera: Gelechiidae) em tomateiro. N eotropical Entomology, v.30, n.3, p.455459, 2001.

LhamBY, J.C.B.; BACALTCHUK, B. Informações técnicas para a safra 2007 trigo e triticale. Passo Fundo: Embrapa Trigo, 2007. 74p. (Embrapa Trigo: Documentos, 69).

CREDLAND,P.Thestructureof bruchid eggsmay explainthe ovicidal effect of oils.) ournal of Stored P roducts R esearch, v.28, n.5, p.1-9, 1992.

Cruz, I.; Vendramim, J.D. Biologia do pulgão-verde em diferentes hospedeiros. Pesquisa A gropecuária Brasileira, v.3, n.24, p.277-282, 1989.

FERREIRA, D.F. A nálisesestatísticaspor meio doSisvar para Windows versão 4.0. In: REUNIÃO ANUAL DA REGIÃO BRASILEIRA DA SOCIEDADE INTERNACIONAL DE BIOMETRIA , 45., 2000, São Carlos.A nais. São Carlos: UFSCar, 2000. p.255-258. 
Gravena, S. O controle biológico na cultura algodoeira. Informe A gropecuário, v.9, n.1, p.3-15. 2003.

GRIPWALL,E. Theeffectof a neem-based insecticideon three important greenhouse pests. Integrated Control in Glasshouses. IO BC Bulletin, v.22, n.1, p.97-100, 1999.

HASSAN, S.A. Métodos padronizados para testes de seletividade, com ênfase em Trichogramma. In: PARRA, J.R.P.; Zucchi, R.A. (Eds.) Trichogramma e o controle biológico aplicado. Piracicaba: FEA LQ, 1997. p.207-234.

IPERTI, G. Biodiversity of predaceous coccinellidae in relation to bioindication and economic importance. A griculture, Ecosystems and Environment, v.74, p.323342, 1999.

IZAWA, Y.; UChIDA, M.; SUgImoto, T.; A sal, T. Inhibition of chitin biosynthesis by buprofezin analogs in relation to their activity controlling N ilaparvata lugens Stal. Pesticide Biochemistry and Physiology, v.24, n.4, p.343347, 1985.

LoWERY, D.T.; IsMAN, M.B. Toxicity of neem to natural enemies of aphids. Phytoparasitica, v.23, n.4, p.297-306, 1995.

Medina, P.; Budia, F.; Tirry, L.; Smagghe, G.; Vinuela, E. Compatibility of Spinosad, Tebufenozide and Azadirachtin with eggs and pupae of the predator Chrysoperla carnea (Stephens) under laboratory conditions. Biocontrol Scienceand Technology, v.11, n.5, p.597-610, 2001.

Medina. P.; Smagghe, G.; Budia, F.; Tirry, L.; Vinuela, E. Toxicity and absorption of azadirachtin, diflubenzuron, pyriproxifen, and tebufenozideafter topical application in predatory larvaeofC hrysoperla carnea (Neuroptera: Chrysopidae). Pest M anagement, v.32, n.1, p.196-203, 2003.
Medina, P.; Budia, F.; Del Estal, P.; Vinuela, E. Influence of azadirachtin, a botanical insecticide, on Chrysoperla carnea (Stephens) reproduction: toxicity and ultrastructural approach. Journal of Economic Entomology, v.97, n.1, p.43-50, 2004.

Mordue, A.J.; BLACKWELL, A. Azadirachtin: an update.j ournal of Insect Physiology, v.39, n.8, p.903-924, 1993.

Nathan, S.S.; Kalaivani, K.; Murugan, K.; Chung, P.G. The toxicity and physiological effect of neemlemonoidson Cnaphalocrocis medinalis (Guenée) the rice leaffolder. PesticideBiochemistry and Physiology,v.81,p.113-112,2005.

REIS, P.R. A spectos bioecológicos eseletividade de agroquímicos a Iphiseiodes zuluagai Denmark \& M uma, 1972 (A cari: Phytoseiidae). 1996. 154p. Tese (Doutorado em Entomologia)-EscolaSuperior deA gricultura“Luiz de Queiroz"-UniversidadedeSão Paulo, Piracicaba, 1996.

Scotr, A.J.; KNotr, M.A. A cluster analyses method for grouping meansintheanalyses of variance.Biometrics, v.30, n.3, p.507-512, 1974.

VENDRAMIM,J.D. Uso deplantas inseticidas no controlede pragas. In:CICLODEPALESTRASSOBREAGRICULTURA ORGÂNICA. 2., 1997, São Paulo. A nais. São Paulo: Fundação Cargil, 1997. p.64-69.

Vogt, H.; Gonzalez, M.; Adan, A.; Smagghe, G.; Vinuela, E. Efectos secundarios de la azardiractina, vía contacto residual, en larvasjóvenes del depredadorChrysoperla carnea (Stephens) (Neuroptera, Chrysopidae). Boletín de Sanidad V egetal de Plagas, v.24, n.1, p.67-78, 1998.

Recebido em 3/ 7/ 06

A ceito em 3/ 9/ 07 